\title{
EigenFairing: 3D Model Fairing using Image Coherence
}

\author{
Pragyana Mishra, Omead Amidi, and Takeo Kanade \\ Robotics Institute, Carnegie Mellon University \\ Pittsburgh, Pennsylvania 15213, USA
}

\begin{abstract}
A surface is often modeled as a triangulated mesh of 3D points and textures associated with faces of the mesh. The 3D points could be either sampled from range data or derived from a set of images using a stereo or Structurefrom-Motion algorithm. When the points do not lie at critical points of maximum curvature or discontinuities of the real surface, faces of the mesh do not lie close to the modeled surface. This results in textural artifacts, and the model is not perfectly coherent with a set of actual images - the ones that are used to texture-map its mesh.

This paper presents a technique for perfecting the 3D surface model by repositioning its vertices so that it is coherent with a set of observed images of the object. The textural artifacts and incoherence with images are due to the non-planarity of a surface patch being approximated by a planar face, as observed from multiple viewpoints. Image areas from the viewpoints are used to represent texture for the patch in eigenspace. The eigenspace representation captures variations of texture, which we seek to minimize.

A coherence measure based on the difference between the face textures reconstructed from eigenspace and the actual images is used to reposition the vertices so that the model is improved or faired. We refer to this technique of model refinement as EigenFairing, by which the model is faired, both geometrically and texturally, to better approximate the real surface.
\end{abstract}

\section{Introduction}

A model of a real surface is often represented as a textured mesh of 3D points. The points form vertices of a triangulated mesh, faces of which are texture-mapped to model the surface. This representation has proven to be effective, as a significant number of surfaces can be modeled by a mesh of planar faces. Most sensed 3D data, however, do not always lie at critical points of maximum curvature or discontinuities, such as corners and edges of the real surface [Figure 1]. Laser scanned data misses out on these strategic but spatially minute points. Features selected and tracked using local pixel intensities in images for stereo or SfM algorithms do not always correspond to points of discontinuity of the observed surface. These facts lead to a poor approximation of the surface by planar faces, and result in not-so-perfect appearance of modeled surfaces. This paper brings out this salient aspect of 3D modeling. It presents a new method of repositioning vertices of the mesh to those critical points for an improvement in the geometrical and textural 

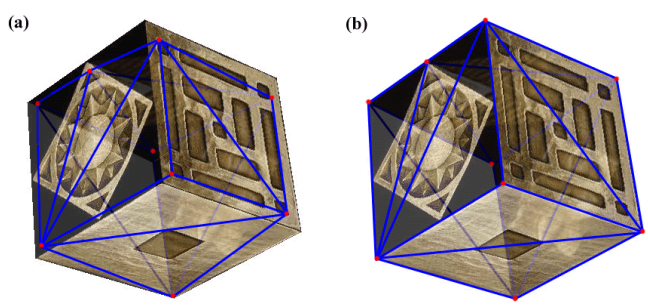

Figure 1: (a) 3D points used for creating the model do not lie at geometrical extremities or points of discontinuity of the cube's surface. Faces of the mesh with such 3D points as vertices are poor approximation of the real surface. (b) For the new vertex positions, the mesh faces lie on the surface of the cube, thus creating a good model.

quality of the model. We show that this seemingly incremental change has a significant impact on the model's overall appearance.

The 3D model is refined or faired by relocating vertices such that the planar faces lie closer to the surface patches they approximate. As the real surface is not known to us, the same set of images that is used for texture-mapping faces of the mesh guides the fairing process. A mesh face, that approximates a surface patch, is projected into images to yield triangular image areas. A set of these image areas corresponding to the same surface patch has information of how good its planar approximation is. Had the surface patch been planar, the image areas corresponding to its planar approximation would be the same when warped into a fixed triangular area [Figure 2]. As the real world is not built of simplicial elements, the variations of texture in the image areas can be attributed to the non-planarity of the surface patch. We would like to get the best possible representation of texture from the image areas by encoding the textural variations.

Given a set of image areas, a compact representation of these variations can be derived in terms of a small number of orthogonal basis images. This representation, also called an eigenspace decomposition, encodes the minute variations of texture as observed from different viewpoints [7]. We reposition vertices of the mesh such that these textural variations are minimized. In the process, faces of the mesh better approximate the real surface in the geometrical sense. Also, the texture-mapped 3D mesh-the 3D model-appears to be as close as possible to the actual images when observed from the same camera viewpoints. This coherence between the actual image area and the textural representation of the corresponding surface patch can be quantified as the distance-from-eigenspace of the image area. The better a triangular face approximates the surface patch geometrically, the smaller is the variation of appearance in images, and the smaller is the distance-fromeigenspace of the image-areas corresponding to the patch. We refer to this idea of 3D model refinement as EigenFairing.

\section{Related Work}

The problem of model refinement based on image data has been studied extensively. A single image can be used to reposition vertices of a texture-mapped mesh by minimizing error metrics based on the vertex positions, surface normals, and other surface properties 


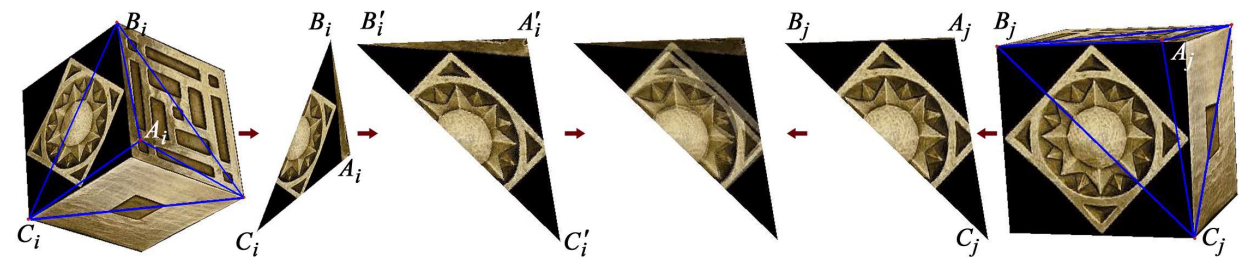

Figure 2: Image areas $A_{i} B_{i} C_{i}$ in the $i$-th image and $A_{j} B_{j} C_{j}$ in the $j$-th image correspond to the same $3 \mathrm{D}$ mesh face. Affine warping of $A_{i} B_{i} C_{i}$ into $A_{j} B_{j} C_{j}$ yields image patch $A_{i}^{\prime} B_{j}^{\prime} C_{j}^{\prime}$. The texture within the two areas, $A_{i}^{\prime} B_{i}^{\prime} C_{i}^{\prime}$ and $A_{j} B_{j} C_{j}$, are not the same because vertex $A$ is not at an edge or a corner, and the mesh face does not lie on the actual 3D surface of the object. Note the ghosting of edges in their superimposition (average) in the center.

such as color and texture [4]. The algorithm never accesses the pixels of the single-image texture and merely updates texture coordinates in the image. Image-driven mesh simplification [5] compares actual images against images of the simplified model to decide which portions of a model to refine through the edge collapse operator. In image-consistent surface triangulation [6], the initial mesh is refined through edge-swaps to best account for observed images. The edge-swapping scheme, that uses the weighted-average of affinewarped image areas, works well only if a surface patch has enough texture and is close to the planar face that approximates it. Other algorithms [3] [8] recover surface shape and reflectance properties from multiple images by deforming a 3D representation. However, these methods optimize complicated objective functions that combine several image and geometric-based constraints. We shall show that a simple objective function, based on the eigenspace representation of texture, can be minimized to refine the geometry of 3D models. Our approach is especially consequential for modeling surfaces whose textural detail is denser than the geometrical level of detail.

Eigenspace texture methods [7] [1] encode appearance variations of a surface patch under various viewing conditions. Appearance of patches are encoded using the eigenspace method, and new views are reconstructed from their eigenspace representations. We shall use an eigenspace representation of dimensionality five to represent texture of 3D surface. It has been shown that for diffuse surfaces of arbitrary texture, the first five components of eigenspace explain most of the image variation [2].

EigenFairing is similar to the multiresolution surface reconstruction algorithm [9], but it does not attempt to subdivide the mesh to account for perspective effects. It refines a given mesh to best approximate an observed surface. The planarity of a surface patch as compared to a mesh face that models it, i.e. perspective distortion, depends on the position of the vertices. One of the critical components of model refinement, that seems to be missing in previous work, is the relocation of vertex points so that they lie at the extremities or critical points of object geometry.

\section{Image Coherence}

If a surface patch is not planar and has perspective distortion, how can texture of its planar approximation - the mesh face-be best represented, given the triangular pixel-areas in 


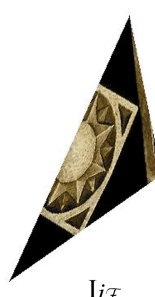

$\mathrm{I}_{i \mathcal{F}}$

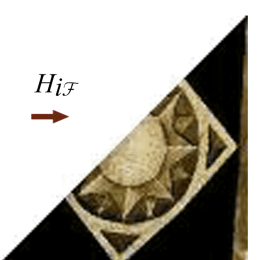

IiF

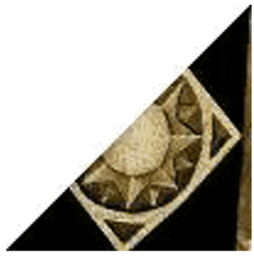

$U_{\exists} \mathbf{c}_{i}$

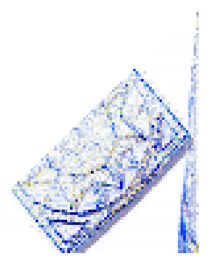

$\rho\left(I_{i \mathcal{F}}-U_{\mathcal{F}} \mathbf{c}_{i}\right)$

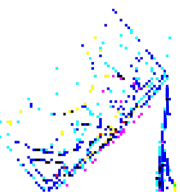

$\rho>e_{T}$

Figure 3: The triangular image patch $\mathrm{I}_{i \mathscr{F}}$ in the $i$-th image corresponding to face $\mathscr{F}$ is warped to a fixed cell image $I_{i \mathscr{F}}$. The reconstructed cell image using the first five components of eigen-space is $U_{\mathscr{F}} \mathbf{c}_{i}$. The Geman-McClure norm between $I_{i \mathscr{F}}$ and $U_{\mathscr{F}} \mathbf{c}_{i}$ for $\sigma=100$, and the spatial distribution of outliers $\left(e_{T}=\sigma / \sqrt{3}\right)$ are on the right.

multiple images that correspond to it? The triangular image areas depend on viewpoints of the camera as well as degree of non-planarity of the 3D-surface patch. The affine warping of triangular image areas into each other does not align the texture within [Figure 2]. Estimating texture that corresponds to the mesh face by weighted-average of pixels from different images [6] leads to blurring of the estimated texture. Since image-consistency is sensitive to textural variations, averaging of affine-warped image areas leads to complicated overlapping surfaces and chances of the refinement algorithm being trapped in local minima. Moreover, textural variations of image areas corresponding to the same 3D-surface patch can be exploited for refining the mesh. Image coherence considers this intra-image textural variation, as well as consistency of estimated texture when compared to actual images.

For a given set of image areas corresponding to a single 3D-surface patch, image coherence constructs a small set of basis images that best captures the variations in texture. These basis images form a view-based representation of the texture of the patch. Each triangular image area is affine warped to a fixed triangular area called the cell image [7]. Since each cell image is created by affine warping the triangular image areas corresponding to a patch, the number of basis vectors to adequately represent its appearance depends on the planarity of the patch. This is valid under the assumption that the patch has Lambertian reflective properties. The more planar a patch is, the better is its approximation by the mesh face, the lower is its perspective distortion as seen from the image-viewpoints, and therefore, the lower is the number of basis vectors needed to represent the texture of the patch. Considering the first $k$ principal components corresponding to the $k$ largest singular values, the basis image-set is $\boldsymbol{U}_{\mathscr{F}}=\left[U_{\mathscr{F} 1}, U_{\mathscr{F} 2}, \ldots, U_{\mathscr{F} k}\right]$ for face $\mathscr{F}$. Let $U_{\mathscr{F}} \mathbf{c}_{i}$ denote the reconstructed cell image corresponding to the $i^{t h}$-image patch. The set of scalar values, $\mathbf{c}_{i}=\left[c_{i 1}, c_{i 2}, \ldots, c_{i k}\right]$, is computed by taking the dot product of the cell image and basis images $\boldsymbol{U}_{\mathscr{F}}$. Thus, $U_{\mathscr{F}} \mathbf{c}_{i}$ is constructed by a linear combination of the $k$ basis images; $U_{\mathscr{F}} \mathbf{c}_{i} \equiv \sum_{m=1}^{k} c_{i m} U_{\mathscr{F} m}$.

An image coherent representation is the best possible approximation, $U_{\mathscr{F}} \mathbf{c}_{i}$, over all image areas, i.e., for $i=1,2, \ldots, n$. For a Lambertian surface, such a representation reflects the accuracy of approximation of the textured patch by the mesh geometrical element. Image coherence is based on the closeness of these two approximations: the textural approximation as captured by the basis images, and the geometrical approximation of the 
physical 3D patch by the planar face. Minimizing the error in representation of surface texture minimizes the error in the approximation of the surface patch by a linear mesh element.

\section{Model Fairing}

Given a set of basis texture-images $\boldsymbol{U}_{\mathscr{F}}$ corresponding to a face $\mathscr{F}$, we reconstruct the image patch $U_{\mathscr{F}} \mathbf{c}_{i}$ in the $i$-th image. The objective function to be minimized for the set of $n$ cell images, $I_{i \mathscr{F}} ; i=1, \ldots, n$, is

$$
E(\mathbf{c})=\sum_{i=1}^{n} \sum_{\boldsymbol{u}_{\mathscr{F}}} \rho\left(I_{i \mathscr{F}}(\boldsymbol{u})-\left[U_{\mathscr{F}} \mathbf{c}_{i}\right](\boldsymbol{u})\right)
$$

The error norm $\rho$ defined over residual pixel-error in cell images. $\boldsymbol{u}_{\mathscr{F}}$ is the coordinate of cell pixels. Instead of exhaustively searching around each vertex in $3 \mathrm{D}$ space, we formulate an iterative search method.

\subsection{Vertex Displacement}

Let $\eta=\left[\begin{array}{lll}\eta_{X} & \eta_{Y} \eta_{Z}\end{array}\right]^{T}$ represent a 3D displacement of a vertex. The faired vertex point $\tilde{\mathbf{x}}_{\mathscr{V}}$ is updated as $\tilde{\mathbf{x}}_{\mathscr{V}} \leftarrow \mathbf{x}_{\mathscr{V}}+\eta$. The goal is to simultaneously find the coefficients $\mathbf{c}$ and displacement vector $\eta$ that minimize the objective function of the residual error;

$$
E(\mathbf{c}, \eta)=\sum_{i=1}^{n} \sum_{\tilde{\boldsymbol{F}_{\tilde{F}}}} \rho\left(I_{i \tilde{\mathscr{F}}}(\boldsymbol{u})-\left[U_{\tilde{\mathscr{F}}} \mathbf{c}_{i}\right](\boldsymbol{u})\right)
$$

for the faces $\tilde{\mathscr{F}}$ constructed from the new vertices $\tilde{\mathbf{x}}_{\mathscr{V}}$. This optimization interleaves two sub-problems. The first sub-problem is to minimize $E(\mathbf{c}, \eta)$ with respect to $\mathbf{c}$ while the vertex $\mathbf{x}_{\mathscr{V}}$ is kept fixed. This is the same as the eigen-texture method [7] discussed in the last section.

The second sub-problem is to minimize $E(\mathbf{c}, \eta)$ with respect to the fairing parameters or displacement $\eta$, this time with the coefficients $\mathbf{c}$ held fixed. The image patch $I_{\mathscr{F}}$ corresponding to face $\mathscr{F}$ gets warped to $I_{\tilde{F}}$ that corresponds to a new face $\tilde{\mathscr{F}}$. For a given set of basis images $\boldsymbol{U}_{\mathscr{F}}$, we have to determine a new set of image patches and corresponding cell images, $I_{i \tilde{\mathscr{F}}}(\boldsymbol{u})$ for $i=1,2, \ldots, n$, such that the following is minimized:

$$
\sum_{i=1}^{n} \sum_{\tilde{\boldsymbol{F}}} \rho\left(I_{i \tilde{\mathscr{F}}}(\boldsymbol{u})-\left[U_{\mathscr{F}} \mathbf{c}_{i}\right](\boldsymbol{u})\right)
$$

Due to a vertex displacement $\eta$, cell pixels at $\boldsymbol{u}$ get displaced to new cell image coordinates as $\boldsymbol{u}+v_{i \mathscr{F}}(\boldsymbol{u}, \eta)$. For a given pixel-coordinate displacement function $v_{i \mathscr{F}}(\boldsymbol{u}, \eta)$, the new cell image is $I_{i \tilde{\mathscr{F}}}(\boldsymbol{u})=I_{i \mathscr{F}}\left(\boldsymbol{u}+v_{i \mathscr{F}}(\boldsymbol{u}, \eta)\right)$. Ideally, we should have

$$
I_{i \mathscr{F}}\left(\boldsymbol{u}+v_{i \mathscr{F}}(\boldsymbol{u}, \eta)\right)=\left[U_{\mathscr{F}} \mathbf{c}_{i}\right](\boldsymbol{u})
$$

Equation (4) states that there are pixel displacements $v_{i \mathscr{F}}(\boldsymbol{u}, \eta)$ that when applied to the image patch $I_{i \mathscr{F}}$ make $I_{i \mathscr{F}}$ look like some image reconstructed from the eigenspace. A first order Taylor series expansion of the left hand side of Equation (4) yields

$$
I_{i \mathscr{F}}(\boldsymbol{u})+\nabla I_{i \mathscr{F}} \cdot v_{i \mathscr{F}}(\boldsymbol{u}, \eta)=\left[U_{\mathscr{F}} \mathbf{c}_{i}\right](\boldsymbol{u})
$$


Summing the residual pixel-error $e_{c}$ over cell pixels $\boldsymbol{u}_{\mathscr{F}}$ corresponding to face $\mathscr{F}$ in all images, the error function can be written in terms of $e_{c}$ as

$$
E(\mathbf{c}, \eta)=\sum_{i=1}^{n} \sum_{\boldsymbol{u}_{\mathscr{F}}} \rho\left(e_{c}\right) \quad \text { where } \quad e_{c} \equiv \nabla I_{i \mathscr{F}} \cdot v_{i \mathscr{F}}(\boldsymbol{u}, \eta)+\left(I_{i \mathscr{F}}(\boldsymbol{u})-\left[U_{\mathscr{F}} \mathbf{c}_{i}\right](\boldsymbol{u})\right)
$$

\subsection{Optimization}

The minimization of $E(\mathbf{c}, \eta)$ with respect to $\eta$ can be obtained using the Gauss-Newton algorithm. In the Gauss-Newton method, a search direction is computed using the gradient, and a first-order approximation to the Hessian for the given objective function $E(\mathbf{c}, \eta)$. The $k$-th element of gradient vector $\mathbf{g}$ is

$$
\mathbf{g}_{k}=\sum_{i=1}^{n} \sum_{\boldsymbol{u}_{\mathscr{F}}} \dot{\rho}\left(e_{c}\right) \frac{\partial e_{c}}{\partial \eta_{k}}, \quad \frac{\partial e_{c}}{\partial \eta_{k}}=\left(\nabla I_{i \mathscr{F}} \cdot \frac{\partial v_{i \mathscr{F}}(\boldsymbol{u}, \eta)}{\partial \eta_{k}}\right) \quad \text { for } \quad k \in\{X, Y, Z\} .
$$

where $\dot{\rho}$, also called the influence function, is the derivative of the error norm $\rho$ with respect to the residual pixel error. We have chosen the Geman-McClure norm as our error norm $\rho$; it is defined over the residual pixel-error $e_{c}$ in cell images. Given a scale factor $\sigma$ that controls the convexity of the norm and its influence to outliers, we have: $\rho\left(e_{c}\right)=e_{c}^{2} /\left(\sigma+e_{c}^{2}\right), \dot{\rho}\left(e_{c}\right)=2 \sigma e_{c} /\left(\sigma+e_{c}^{2}\right)^{2}$, and $\ddot{\rho}\left(e_{c}\right)=2 \sigma\left(\sigma-3 e_{c}^{2}\right) /\left(\sigma+e_{c}^{2}\right)^{3}$. The $\{k, l\}$-th element of the Hessian $\mathbf{H}$ is

$$
\mathbf{H}_{k l}=\sum_{i=1}^{n} \sum_{\mathscr{u _ { F }}} \ddot{\rho}\left(e_{c}\right) \frac{\partial e_{c}}{\partial \eta_{k}} \frac{\partial e_{c}}{\partial \eta_{l}} \quad \text { for } \quad k, l \in\{X, Y, Z\}
$$

The objective function $E$ is convex when the Hessian $\mathbf{H}$ of $E$ is positive definite. A positive definite Hessian indicates that the function has a unique optimum, in the local neighborhood, whereas a Hessian that has one or more eigenvalues zero will allow an entire manifold of solutions to minimize the objective function. $E$ is locally convex when $\ddot{\rho}\left(e_{c}\right)>0 \forall e_{c}$. For small $\sigma$ values, $\ddot{\rho}\left(e_{c}\right)$ can be negative, and therefore, one may not get a descent direction. As $\dot{\rho}(0)=0, \ddot{\rho}\left(e_{c}\right)$ is substituted by its secant approximation, $\dot{\rho}\left(e_{c}\right) / e_{c}$, for small values of $e_{c}$, and is positive everywhere. Substituting in equation 8 ,

$$
\sum_{l \in\{X, Y, Z\}}\left(\sum_{i=1}^{n} \sum_{\boldsymbol{u}_{\mathscr{F}}} \frac{\dot{\rho}\left(e_{c}\right)}{e_{c}} \frac{\partial e_{c}}{\partial \eta_{k}} \frac{\partial e_{c}}{\partial \eta_{l}}\right) \delta \eta_{l}=-\sum_{i=1}^{n} \sum_{\mathscr{\boldsymbol { u } _ { F }}} \dot{\boldsymbol{\rho}}\left(e_{c}\right) \frac{\partial e_{c}}{\partial \eta_{k}} \text { for } k \in\{X, Y, Z\} .
$$

The fairing displacements, $\eta=\left[\eta_{X} \eta_{Y} \eta_{Z}\right]^{T}$, are iteratively updated for step $m$ as $\eta^{(m+1)}=$ $\eta^{(m)}+\delta \eta$ and $\eta^{(0)}=\left[\begin{array}{lll}0 & 0 & 0\end{array}\right]^{T}$. After each vertex fairing step, the image patches are updated by considering the new position of the vertex. The new image patches are warped into their corresponding cell images. These cell images are used for the next optimization step. As the warping registers the image patches and the eigenspace, the approximation $\left[U_{\mathscr{F}} \mathbf{c}_{i}\right]$ continues to improve and the texture representation gets better.

\subsection{Cell-Pixel Displacement}

The cell-pixel displacement function $v_{i \mathscr{F}}(\boldsymbol{u}, \eta)$ in the $i$-th image, corresponding to the face $\mathscr{F}$, is related to the vertex-displacement $\eta$. The $3 \times 4$ projection matrix $\mathbf{P}$ for the $i$-th 

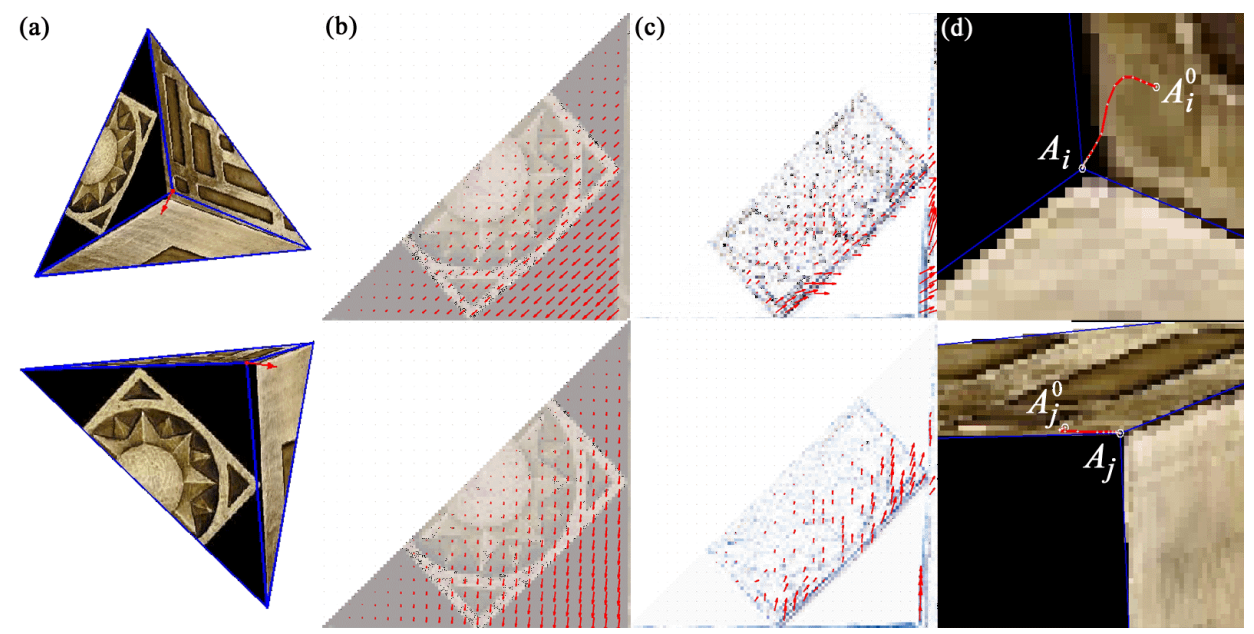

Figure 4: (a) A vertex displacement induces pixel displacements $\delta \mathbf{u}_{i \mathscr{V}}$ and $\delta \mathbf{u}_{i \mathscr{V}}$, shown as red arrows, in image $i$ and $j$. (b) Pixel displacements are used to calculate the cell pixeldisplacements $v_{i \mathscr{F}}$ and $v_{j \mathscr{F}}$ for the $i$-th and $j$-th cell images. (c) Optical flow calculated using constant brightness constraint between the cell image and its eigenspace reconstruction is superimposed on their difference. (d) The path of successive vertex displacements. $A_{i}^{0}$ and $A_{j}^{0}$ correspond to the initial position of the vertex. $A_{i}$ and $A_{j}$ correspond to the $3 \mathrm{D}$ point that the vertex converges to. (All red arrows are displacements magnified $10 \times$ )

image is known, and let its $p$-th row be represented as $\left[\mathbf{r}_{p} \mid t_{p}\right]$ for $p=1,2$ and 3. The pixel displacement in the $i$-th image corresponding to a small 3D vertex displacement, $\eta$, is

$$
\delta \mathbf{u}_{i \mathscr{V}}=\left[\begin{array}{ll}
\frac{\left(\mathbf{r}_{3} \mathbf{x}+t_{3}\right) \mathbf{r}_{1} \eta-\left(\mathbf{r}_{1} \mathbf{x}+t_{1}\right) \mathbf{r}_{3} \eta}{\left(\mathbf{r}_{3} \mathbf{x}+t_{3}\right)^{2}} & \frac{\left(\mathbf{r}_{3} \mathbf{x}+t_{3}\right) \mathbf{r}_{2} \eta-\left(\mathbf{r}_{2} \mathbf{x}+t_{2}\right) \mathbf{r}_{3} \eta}{\left(\mathbf{r}_{3} \mathbf{x}+t_{3}\right)^{2}}
\end{array}\right]^{T}
$$

This pixel displacement causes a change in the pixels of the cells. For pixels corresponding to face $\mathscr{F}$ in the $i$-th image, the affine transformation $H_{i \mathscr{F}}$ (a $2 \times 3$ matrix) between the image patch and its cell is known, and $\boldsymbol{u}=H_{i \mathscr{F}}\left[\begin{array}{ll}\mathbf{u}^{T} & 1\end{array}\right]^{T}$. The cell-pixel displacement function can now be related to the vertex pixel displacement in the image as

$$
v_{i \mathscr{F}}\left(\boldsymbol{u}_{\mathscr{V}}, \eta\right)=H_{i \mathscr{F}}\left[\begin{array}{ll}
\delta \mathbf{u}_{i \mathscr{V}}^{T} & 0
\end{array}\right]^{T}+\delta H_{i \mathscr{F}}\left[\begin{array}{ll}
\mathbf{u}_{i \mathscr{V}}^{T} & 1
\end{array}\right]^{T}
$$

For small pixel displacements, $\delta H_{i \mathscr{F}}$ is usually negligible. Once the cell-pixel displacement at the moving vertex is determined, cell-pixel displacements over the entire cell are calculated by interpolation, because the displacements at the other two fixed vertices and along their connecting edge are zero. The goal is to achieve cell-pixel displacements that vary linearly across the cell image from the moving vertex to the other two vertices, or are as close as possible to a linear variation. We are trying to determine the location of the faired vertex that best approximates the surface patch with a planar face of the mesh. The same vertex location yields observed image-pixel displacements that are closest to linear variation of image-pixel displacements of a planar face. For the new position of the vertex, the cell-pixel displacements lead to a better approximation of the surface patch; hence, a more linear variation of flow or pixel displacements appears across the cell image. 


\section{An Illustration}

At first, we illustrate an EigenFairing process for a unit cube with textures on three faces visible in 12 images. The vertex $A$, common to the three faces as shown in Figure 2, is faired such that it corresponds to the actual corner of the cube. The cell size is $128 \times 128$ pixels. The path of the vertex, as it moves towards the actual corner, is displayed in Figure 4(d) for two views. A vertex displacement during the fairing process is shown in Figure 4(a). The interpolated pixel-displacements in the cell images, for the same vertex displacement, are shown in Figure 4(b). Figure 4(c) shows the optical flow fields computed between the cell image and its eigenspace reconstruction. They point in the opposite direction to the pixel-displacements. The estimated vertex displacement is the change in vertex position for which induced pixel-displacements on faces best counterbalance flow-fields between the cell-images and their eigenspace reconstructions.

\section{Results with Real Data}

We applied EigenFairing to real outdoor scenes. An initial set of 3D points for generating the mesh was sampled from range data at points of geometrical extremities and discontinuities, as shown in Figure 5(a). The data was registered with a sequence of 22 images, two of which are shown in Figure 5, by selecting the closest points of correspondence. The points do not accurately correspond to physical corners in images, and edges of the mesh are not aligned with edges of the facade, as viewed in the images. Notice the textural artifacts if this mesh is used for modeling the facade, in Figures 5(a, left column) and 6(a). After EigenFaring the initial model, the 3D vertices correspond to actual corners, and edges of the mesh are aligned with physical edges in images [Figure 5(b)]. Also, the facade is better modeled in a geometrical sense and, at the same time, devoid of textural artifacts [Figure 6(b)]. Figure 7 shows another real data set where a SfM algorithm was used to derive the initial position of vertices from 10 images. For both data sets, the initial meshes are generated using Image-consistent Triangulation [6].

The robustness of the fairing process was increased by adding a coarse-to-fine refinement strategy. A five-level pyramid was used where $\sigma_{\text {smooth }}$ varied linearly from 6.0 to 1.2. The Geman-McClure parameter was chosen as $\sigma=\max \left(\left|e_{c}\right|\right) / \sqrt{3}$. The error norm minimizes the effect of outliers that usually appear at geometrical edges of image areas, specularitites, or due to large textural discontinuities within a patch [Figure 3]. The neighborhood of 3D points for which the initial vertex converges to the faired position depends on how textured the neighboring faces are and how well their textures are represented in eigenspace. The new 3D point may not always lie on the actual surface, although its neighboring faces collectively represent the actual surface region with greater accuracy, both geometrically and texturally.

\section{Conclusion}

The EigenFairing algorithm refines a texture-mapped mesh to better represent a surface by relocating vertices of the mesh. The relocation process is carried out such that the texture mapped onto the faces is best represented, and the resulting 3D model is coherent with observed images. Coherence of texture-mapped faces with images has been formulated 

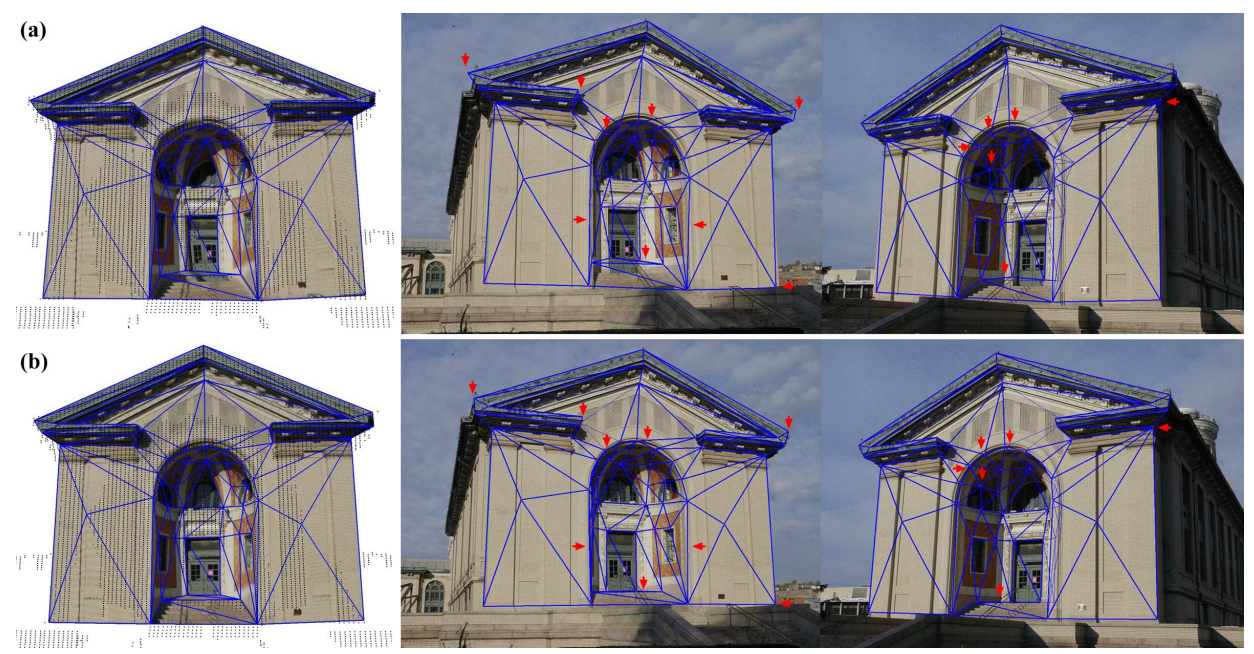

Figure 5: On the first column, 3D range data is superimposed on the texture-mapped models before and after EigenFairing. Range scans often miss out corners due to occlusions or their spatial minuteness. Selected vertices are not always best positioned for surface approximation, as seen on the inner dome. The edges are close to, but not perfectly aligned with the physical edges of the building. Row (a) Initial mesh. Row (b) The EigenFaired mesh. The red arrows point at mesh elements that have considerably improved the model.
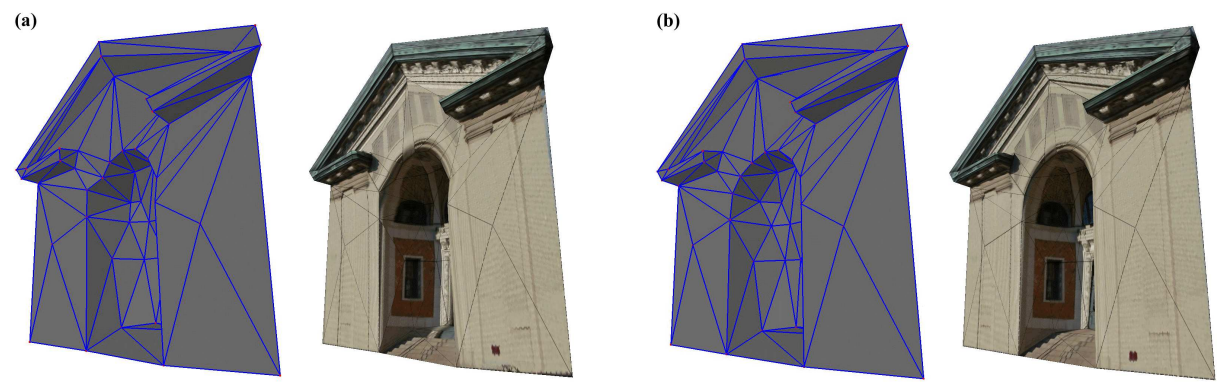

Figure 6: (a) The mesh and the texture-mapped model for the initial position of vertices. (b) The EigenFaired mesh and model. Notice that the initial mesh has irregularities in structure along the arch and on the inner dome. The corresponding textural artifacts are clearly visible in the model. The EigenFaired mesh better approximates the geometrical structure of the building. Its texture-mapped model is devoid of artifacts at the same time.

as minimizing the distance between observed textures corresponding to the faces of the mesh and their eigenspace reconstructions. We have shown that minimizing this distance leads to a better geometrical approximation of the unknown surface by the $3 \mathrm{D}$ model. EigenFairing couples geometrical properties of a 3D model with its textural properties or albedo. Coherence of texture leads to a faired mesh that is physically closer to the true surface. This approach is significant for modeling a surface whose textural variation is much higher than the geometrical resolution required to represent the surface. 

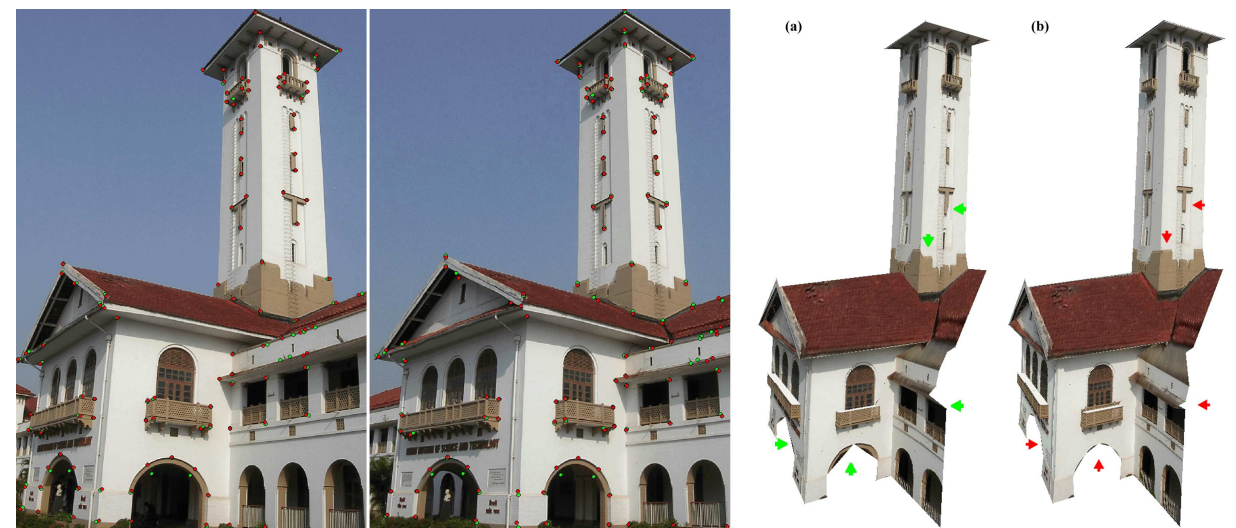

Figure 7: On the left are two images showing the initial vertex positions in green and the faired vertices in red. The faired vertices accurately correspond to corners and edges of the architecture. Notice the points along the archways, roof and balcony edges. The initial model and the EigenFaired model are shown in (a) and (b) respectively. The green and red arrows point out the presence and absence of modeling artifacts respectively.

\section{References}

[1] T.F. Cootes, G.J. Edwards, and C.J. Taylor. Active appearance models. IEEE Transactions on Pattern Analysis and Machine Intelligence, 23(6):681-685, June 2001.

[2] R. Epstein, P. Hallinan, and A. Yuille. 5 \pm 2 eigenimages suffice: An empirical investigation of low-dimensional lighting models. IEEE Workshop on Physics-based Modeling in Computer Vision, pages 108-116, 1995.

[3] P.V. Fua and Y.G. Leclerc. Taking advantage of image-based and geometry-based constraints to recover 3-D surfaces. Computer Vision and Image Understanding, 64(1):111-127, July 1996.

[4] M. Garland and P. S. Heckbert. Simplifying surfaces with color and texture using quadric error metrics. In Proc. of the Conf. on Visualization, pages 263-269, 1998.

[5] P. Lindstrom and G. Turk. Image-driven simplification. ACM Transactions on Graphics, 19(3):204-241, 2000.

[6] D. D. Morris and T. Kanade. Image-consistent surface triangulation. In Computer Vision and Pattern Recognition, volume 1, pages 332-338, June 2000.

[7] K. Nishino, Y. Sato, and K. Ikeuchi. Eigen-texture method: Appearance compression and synthesis based on a 3D model. PAMI, 23(11):1257-1265, November 2001.

[8] S. Nobuhara and T. Matsuyama. Dynamic 3D shape from multi-viewpoint images using deformable mesh models. In Proc. of 3rd International Symposium on Image and Signal Processing and Analysis, pages 192-197, September 2003.

[9] L. Zhang and M. Seitz. Image-based multiresolution modeling by surface deformation. In Technical Report CMU-RI-TR-00-07. Carnegie Mellon University, 2000. 